\title{
Políticas y condiciones laborales del sector docente en Costa Rica
}

\section{Policies and working conditions of the educational sector in Costa Rica}

\author{
Luis Carlos Morales Zúñiga \\ luis23m@gmail.com \\ Universidad de Costa Rica
}

Recibido: 23 mayo 2011 Aceptado: 23 mayo 2011 Corregido: 27 de junio 2011

\section{Resumen}

Este ensayo aborda desde la perspectiva sociológica y sobre todo desde algunos elementos analíticos de sociología de la educación y sociología del trabajo, determinados aspectos de la política educativa nacional que se encuentra vigente, específicamente, aquella relacionada con las condiciones laborales de los docentes. Se plantea la relación que existe entre las condiciones laborales de los y las educadoras y una política de Estado paliativa en relación a algunos problemas educativos, pero que no logra concebir una reforma integral al sistema educativo, de lo cual se sigue la imposibilidad de pensar en un cambio social, y la legitimación de las políticas que rigen el sistema educativo actual. El énfasis del análisis sobre condiciones laborales está en el tema salarial, el interinazgo, y la sobrecarga de trabajo para los docentes.

Palabras clave: sistema educativo, politica educativa, trabajo, sociología, docentes

\begin{abstract}
This essay approaches from a sociological perspective and especially from some analytical elements of sociology of education and sociology of work, certain aspects of the national education current policy, specifically, those related to working conditions of teachers. This paper raises the relationship between working conditions for teachers and State policy in relation to national educational problems, policies that are unuseful to achieve a reform of the education system, as a result of the impossibility of think about social change, and legitimating of educational policies in the current education system. The emphasis of the analysis of working conditions is the subject of salaries, the job instability, and the workload for teachers.
\end{abstract}

Keywords: education system, educational policy, work, sociology, teachers 


\section{Introducción}

En la coyuntura actual existen algunos cambios de enorme interés para la reflexión sobre la política educativa nacional, específicamente sobre las condiciones laborales de los y las docentes, una reflexión que en este ensayo, realizaremos a partir de la sociología de la educación y del trabajo. Estos cambios se han desarrollado en los últimos cinco años, durante la segunda administración del Dr. Oscar Arias Sánchez (2006-2010) y el primer año de la administración de Laura Chinchilla (2010-2011), gobiernos en los que la gestión del Ministerio de Educación Pública (MEP) ha estado a cargo de Leonardo Garnier Rímolo, quien junto al Poder Ejecutivo, ha desarrollado una serie de estrategias de política educativa, las cuales han tenido algunas implicaciones sobre el sistema educativo nacional.

Pero estas implicaciones no han sido integrales, sino que, su alcance es focalizado, y sujeto a distintas interpretaciones, una de ellas es la que plantea el Tercer Informe sobre el Estado de la Educación (CONARE, 2011), una interpretación legitimante ${ }^{1}$, desde la perspectiva que Coraggio (1996) plantea para la clasificación de los tipos investigación educativa.

Estas implicaciones son de difícil captación objetiva, pues no es mucha la investigación existente en este momento, sobre el impacto de la mayoría de las políticas educativas implementadas hasta acá. El Tercer Informe sobre el Estado de la Educación (CONARE, 2011) recoge una serie de investigaciones en el campo educativo, y presenta un balance sobre la implementación de algunas de estas políticas educativas. Sin embargo, este balance tiene el problema de ser una medición estadística a gran escala que puede ser interpretada de muy diversas maneras. Además, el informe llega a algunas conclusiones alarmantes, sobre todo aquella que indica que una reforma integral al sistema educativo en este momento es inviable, y que lo único que puede hacerse es concentrarse en aspectos o necesidades muy especificas del sistema, con lo cual, cualquier cambio sustancial queda descalificado y cancelado a priori (CONARE, 2011).

Por tanto, dado tal contexto, en este ensayo se presenta una visión interpretativa y crítica sobre algunos puntos medulares de la política educativa nacional puesta en marcha durante la gestión del Ministro Leonardo Garnier, desde 2006 hasta 2011, específicamente en lo que concierne a las condiciones laborales de los docentes (todo ello en un marco 
socio-histórico mayor, el cual le da sentido a estas acciones de política educativa). Se analizan las condiciones laborales de los docentes en el sector público, pues en el sector privado hay otros elementos diferenciadores tales como distintos incentivos, condiciones de planta física y de recursos así como otros elementos (a pesar de no ser generalizables a todo el sector privado) con los cuales no se cuenta en el sector público.

Se realiza este análisis a partir, fundamentalmente, aunque no exclusivamente, de los datos estadísticos presentes en el Tercer Informe sobre el Estado de la Educación (CONARE, 2011), como evidencia empírica, y desde una perspectiva teórica particular, basada fundamentalmente en elementos analíticos de la sociología de la educación y del trabajo (Morales, 2010), considerando también el pensamiento crítico (Wacquant, 2006).

Como se indicó anteriormente, las políticas educativas que se analizan serán aquellas relacionadas con las condiciones laborales de la clase trabajadora docente. Es interesante observar, que cuando se han planteado políticas educativas para mejorar la educación nacional, en los últimos años, se han tomado en cuenta elementos como la intervención económica directa del Estado mediante el Programa Avancemos, o bien el sistema de promoción y adelanto de materias, ambas, estrategias que desde la óptica oficial del MEP y del Poder Ejecutivo tienen como principal interés eliminar lo que estas instancias denominan deserción escolar ${ }^{2}$, y aumentar la retención de los estudiantes en el sistema educativo nacional, pero en lo que respecta a condiciones laborales de los y las educadoras, el tema se reduce a lo salarial.

De manera que, en el primer apartado se refiere de forma general al contexto histórico-social del país, como una resultante histórica del nuevo estilo nacional de desarrollo $^{3}$ (Rovira, 2004), en el cual se desarrolla y desenvuelve la clase trabajadora docente, con tal de tener un marco contextual que nos permita entender el sentido que tienen las estrategias de política educativa implementadas sobre las cuales se ocupa este ensayo.

Al mismo tiempo, esto constituye un ejercicio de aquello que Wrigth (1994) llamó imaginación sociológica, es decir, la capacidad de vincular los cursos biográficos con la historia, o bien, hacer visible la relación entre estas particularidades a las que nos enfrentamos tanto en nuestra cotidianidad individual o social, y la estructura social en 
movimiento, dos líneas paralelas que solo el punto de vista del investigador o del sujeto puede interrelacionar, pues esta relación no es obvia, ni salta a la vista.

En un segundo apartado se analizan los datos, es decir, la evidencia empírica de la que disponemos en este momento sobre las condiciones laborales docentes, con tal de esbozar sobre estas políticas, una interpretación fundada en algunos principios teóricos de sociología de la educación, del trabajo, y del pensamiento crítico, tratando de sostener y argumentar como tesis fundamental de este documento, que las reivindicaciones salariales que se han otorgado al sector docente, tienen como principal interés crear una ilusión en torno a los principales problemas que enfrenta este sector ocupacional, mas en realidad, son apenas efímeros cambios que no tienen una incidencia directa sobre el sistema educativo como totalidad concreta, y mucho menos sobre la calidad de la educación, e incluso, aun menos, sobre las condiciones laborales de los docentes como fenómeno integral, pues no es esa la intención de los grupos que han accedido al poder del Estado, y tampoco es la recomendación o conclusión a la que llega el Tercer Informe sobre el Estado de la Educación (CONARE, 2011), legitimando en buena medida, la inacción y la política educativa paliativa y contemplativa.

\section{Educación costarricense en un contexto neoliberalmente condicionado}

El momento histórico-social en el que nos movemos es un contexto que se encuentra condicionado fuertemente por una tendencia neoliberal que ha sido causa y efecto (así como dialécticamente se construye la realidad social), de lo que Rovira (2004) ha llamado el nuevo estilo nacional de desarrollo, y que para efectos del fenómeno educativo, o al menos de una parte de este fenómeno, habíamos caracterizado ya en el texto Mercado laboral, educación superior y formación docente en Costa Rica (Morales, 2011), donde el énfasis analítico está en la tensa y dinámica relación que hay entre el contexto del nuevo estilo nacional de desarrollo, desregulación en la formación docente, en términos de cantidad, y la consecuente saturación del mercado laboral con las consecuencias que ello implica. 
En todo caso, para el fenómeno que en este escrito nos ocupa, realizaremos en este apartado, una descripción de los aspectos del nuevo estilo nacional de desarrollo en el que se despliega la política educativa actual, y que le da sentido a las condiciones laborales que enfrenta el sector docente, pues es de esta forma como se pueden comprender, y captar las características y consecuencias de estas condiciones laborales, que si bien no son trascendentales en términos de impacto sobre el sistema educativo, si encierran una forma de pensamiento ideológicamente posicionada que responde y corresponde a un contexto políticamente determinado, en el cual se desarrolla toda una política educativa.

Este contexto, políticamente condicionado y determinado por la corriente de pensamiento neoliberal, inicia su configuración durante la década de 1980, específicamente en el gobierno de Luis Alberto Monge Álvarez (1982-1986), con la firma del primer Programa de Ajuste Estructural, en 1985, y llega a su afianzamiento o consolidación, con la aprobación en referéndum del Tratado de Libre Comercio con Estados Unidos, el 7 de octubre de 2007, pues finalmente, se logra asegurar jurídicamente un estilo de desarrollo caracterizado, entre otras cosas, por la liberalización de la economía, la atracción de inversión extranjera directa, la atracción de empresas de tecnología, la promoción de las exportaciones, y el desarrollo del turismo de enclave, sobre todo en el litoral pacífico del país (Morales, 2010).

El cambio en el estilo nacional de desarrollo que ha experimentado el país, es una transformación de la estructura social, económica, política y cultural, que se ha llevado a cabo por medio de la interacción entre los actores políticos internos, en un marco de condiciones políticas externas, signadas por una tendencia a la liberalización de la economía en toda la región latinoamericana, sobre todo a partir del Consenso de Washington (Vargas, 2003), liberalización iniciada en la década de 1980, y que ha sido revertida en medida relativa, en algunos países de la región como Brasil, Ecuador, Bolivia y Venezuela.

Para el caso de nuestro país, este cambió ha sido llevado a cabo por las élites que han accedido al poder del Estado durante los últimos treinta años. El cambio, como bien lo explica Rovira (2004) ha sido gradual y, por supuesto, ha tenido algunas resistencias de algunos sectores de la sociedad que se oponen al neoliberalismo. Sin embargo, siguiendo la 
propuesta teórica de Milliband (1985), cuando habla de las posibilidades políticas en la sociedad y el Estado Capitalista, indica que "en este contexto la política es por cierto el arte de lo posible. Pero lo posible esta determinado sobre todo por aquello que parece aceptable a la "comunidad de los negocios” (p.147).

Milliband (1985), naturalmente reconoce la existencia de la competencia por el poder del Estado o por la influencia sobre tal poder. Sin embargo, destaca que es una competencia desigual, donde el capital privado goza de una formidable superioridad (como grupo organizado) frente a otros grupos o intereses, particularmente el de los trabajadores, esto debido a su base de poder económico, tal como ha ocurrido en el caso del nuevo estilo nacional de desarrollo de Costa Rica.

El control privado de los recursos industriales, comerciales financieros, culturales y por lo tanto, educativos, ejerce una presión permanente sobre los gobiernos y el Estado. Esto no quiere decir que los gobiernos se encuentran totalmente desvalidos ante el capital, sino que hay una dinámica de demandas y resistencias, en las cuales unas veces prevalece el capital y en otras, la fuerza del Estado. La injerencia de lo que Milliband (1985) llama el poder de veto del mundo de los negocios, es decir, del capital privado, no es absoluto. Sin embargo, es más grande que cualquier otro interés en el marco de las sociedades capitalistas, es decir, también en el contexto de la sociedad costarricense actual, el poder de veto de las clases trabajadores es reducido, incluyendo el sector docente del país.

Por tanto, los gobiernos en la sociedad capitalista, generalmente optan por ganarse la confianza del mundo de los negocios, y no por enfrentarlo, aceptando las limitaciones a su margen de acción política. Además de los grupos o intereses capitalistas nacionales, los gobiernos deben enfrentar el proceso de internacionalización del capital, es decir, la presión y el poder de fuerzas capitalistas extranjeras, tales como gobiernos fuertes que protegen los intereses de sus empresas cuando estas se internacionalizan, así como de organismos que velan por los intereses de los círculos de negocios, tales como el Banco Mundial, el Fondo Monetario Internacional, entre otros. Estos son los factores condicionantes a los que se refería Rovira (2004), los cuales brindan el marco de acción de los factores políticos internos que definen la política social, económica, y por supuesto, la política educativa de nuestro país. 
No obstante, a partir de la Teoría Democrático-Pluralista, los trabajadores organizados constituyen un grupo de interés de la sociedad que se considera igual al poder del capital. Sin embargo, plantea Milliband (1985), esta tesis no toma en cuenta que los y las trabajadoras organizados no poseen ningún arma comparable al poder del capital en la diaria toma de decisiones. El trabajo organizado carece de una firme base de poder económico, por lo cual posee menor potencial de presión respecto al poder del Estado. Esto es lo que ha ocurrido sobre todo con los trabajadores y trabajadoras de la educación, pues su capacidad de organización no solo es limitada, sino que, se enfrentan a la capacidad de organización del mundo de los negocios, o del sector empresarial que accede al poder del Estado, o bien que presiona de manera privilegiada a aquellos que ejercen el poder de Estado.

Los trabajadores carecen de la presión e influencia que, potencialmente, podrían ejercer instancias extranjeras, como si lo hacen los grupos capitalistas internacionales a favor de los círculos de negocios nacionales, es decir, una instancia como el Fondo Monetario Internacional, o como el Banco Mundial, no tiene su correlato obrerista, no hay ningún sector de la clase trabajadora que tenga una influencia comparable a estos organismos económicos internacionales, lo cual deja en una posición desventajosa a los docentes como sector de la clase trabajadora, en términos de la reivindicación de sus condiciones laborales, y no solo de sus condiciones salariales.

Milliband (1985) destaca también que la principal arma con que cuentan los trabajadores organizados es la Huelga. Sin embargo, ésta requiere de voluntad, decisión, persistencia y determinación por parte de los dirigentes de las organizaciones de trabajadores, de otra forma no es posible que resulte efectiva, puesto que generalmente las acciones huelguistas no cuentan con el apoyo de la población, y ni siquiera de la mayoría de los trabajadores, sino que tienden a presentarse como egoístas, innecesarias, sectoriales, incongruentes con el interés nacional, e incluso criminales. Basta con observar en retrospectiva, las impresiones que da la prensa nacional cuando algunos sectores de los trabajadores de la educación han decidido ir a huelga (Cuvardic, 2005).

Otro problema que enfrentan las organizaciones de trabajadores es la cuestión de la representatividad, pues no siempre los dirigentes logran asumir el papel de representantes 
de todo el sector de los trabajadores, a diferencia de los hombres de empresa que cuando hablan lo hacen, efectivamente, en nombre del círculo de negocios. El sector políticoempresarial, a pesar de no ser un monolito económico ni ideológico, no presenta tantas divisiones como las organizaciones de trabajadores, cuya característica sobresaliente no es la unidad, sino la división, y la falta de cohesión tanto intra, como (naturalmente) intersectorial $^{4}$.

El sector trabajador en cambio, y particularmente el sector docente, como grupo de presión se manifiesta siempre como un interés mucho más seccional que el de los círculos de negocios. Las demandas de las clases trabajadoras generalmente son consideradas en contra del interés nacional, mientras que las de los círculos de negocios se presentan como congruentes con el interés nacional. Esto deriva en una deslegitimación de las demandas del sector trabajador, y sobre todo del sector docente, pues hay una tendencia a restarle importancia a sus reivindicaciones, las cuales, la mayoría de las veces son de carácter salarial, aspecto necesario pero no suficiente para la mejora de las condiciones laborales de los docentes.

Otro de los rasgos sobresalientes del nuevo estilo nacional de desarrollo, de orientación neoliberal, aplicado en nuestro país, es el discurso que aboga por el crecimiento económico por sí mismo, bajo el supuesto de que un país con alto crecimiento económico, permitiría en última instancia, alcanzar un desarrollo social inclusivo, que permita a la mayor parte de la colectividad nacional participar de la bonanza económica, y por lo tanto incrementar el desarrollo social.

Por supuesto, desde esta perspectiva, la educación en general, y particularmente el sector docente se beneficiaría directamente, pues este crecimiento económico alcanzaría a todos los sectores de la clase trabajadora, teoría que ha sido empíricamente descartada para el caso de Costa Rica, por ejemplo, en el análisis que hace Korten (1997) sobre el crecimiento económico experimentado debido a los Programas de Ajuste Estructural en el país; crecimiento que estuvo acompañado de un profundo deterioro de las condiciones sociales de amplios sectores de la población.

En este sentido, Kliksberg (1998), asume una posición crítica interesante frente a lo que se ha denominado la política del derrame, la cual es característica del discurso político 
neoliberal dominante en el nuevo estilo nacional de desarrollo. El supuesto de este planteamiento versa sobre la idea de que el crecimiento macroeconómico deriva en equilibrios económicos y financieros con todos los beneficios que ello conlleva, y que en algún momento de este lógica alcanzaran a los sectores más pobres de la población, y a esta en su conjunto, haciendo alusión a la copa que se rebalsa y se derrama sobre su base. Sin embargo, como bien lo plantea Kliksberg (1998), hay suficiente evidencia empírica como para echar este planteamiento al suelo:

...la evidencia empírica ha demostrado que si bien un elevado crecimiento económico es condición necesaria, no es condición suficiente para reducir la pobreza y la desigualdad social... porque aun habiendo crecimiento, el mismo puede ser con desocupación, con exclusión, sin participación, con debilitamiento de las culturas nacionales, con destrucción del futuro al deteriorarse el medio ambiente. (p.10)

Esto porque, evidentemente, las relaciones entre política, sociedad y economía son de sumo complejas, que pensar en el simplismo de que la riqueza se desbordará desde los más altos estamentos sociales, para llegar a las clases pobres. La revalorización del capital humano y el capital social, es decir, la consideración de otras modalidades de capital no mercantilista, ni natural, los cuales tienen que ver con la calidad de los recursos humanos, y con elementos compartidos como cultura, y diferentes tipos de interrelaciones sociales, podría ser, tal como lo explica Kliksberg (1998) y como lo habían señalado ya Bourdieu y Passeron (2004), podrían ser claves importantes para el desarrollo, las cuales han sido subestimadas en función del capital económico.

La formación del capital humano implica la inversión en áreas como salud, nutrición, educación, innovación tecnológica, investigación científica, entre otras. Y se enfatiza la necesidad de la inversión en educación. Sin embargo, a nuestro modo de ver, las condiciones materiales de una sociedad abren el abanico de las posibilidades culturales y sociales, por lo cual, habría que ver cómo establecer una relación dialéctica entre 
desarrollo económico y desarrollo cultural y social, de manera que el primero posibilite la ampliación del segundo.

No es posible afirmar que exista una relación inversamente proporcional entre crecimiento económico y reducción de la desigualdad, y mucho menos aceptable resulta la antigua tesis de que la inequidad deriva en funcional al proceso de acumulación, es decir, la conceptualización de la inequidad como un mal necesario. Es posible constatar y descartar empíricamente las bondades del mercado y su mano invisible, y observar la creciente desigualdad, exclusión social y pobreza, a pesar de altas tasas de crecimiento económico, un ejemplo claro y clave es Brasil. Esto tema se encuentra relacionado con la cuestión del vinculo entre desarrollo económico y desarrollo social, sobre lo cual Kliksberg (1998) apunta que

...el desarrollo social parece en la experiencia concreta un proceso vital para que pueda existir un desarrollo económico sostenido. Las inversiones en capital humano y social y el mejoramiento de la equidad, además de fines en sí mismos desde la perspectiva de las sociedades democráticas, son necesarias para que el crecimiento económico pueda tener bases firmes. (p. 18)

Es decir, el desarrollo social debe ir de la mano del desarrollo económico, y viceversa, y el tamaño optimo del Estado donde la idea del Estado Regulador está relacionada con las concepciones del neo-institucionalismo, y la Nueva Economía Institucional el cual surge como una relativa sofisticación de la economía neoclásica, pues le agrega un componente más, a saber una serie de instituciones que vendría a dar alguna regularización a la dinámica del mercado. North (1994), define la nueva economía institucional como "el intento de incorporar una teoría de las instituciones a la teoría económica. ...Lo que esta conserva y complementa es el supuesto básico de la escasez y, por ende de la competencia...” (p. 5).

Se argumenta a favor de un abandono de la racionalidad instrumental que hasta hace algunos años, supuestamente, sostenía el modo de acción de la economía neoclásica. En 
este sentido, y siguiendo el planteamiento de North (1994), las instituciones vendrían a dotar de reglas al juego del mercado, serian reguladoras del librecambio, pero no habría una intermediación político-restrictiva importante. En otros términos, el neo-institucionalismo asume las instituciones como entes que regulan a favor -y ahí está lo fundamental- del crecimiento económico. Sin embargo, se dice poco o nada -y esta es la limitación del enfoque- de la distribución social de ese crecimiento, lo cual en última instancia, debería ser el tema central de toda política económica, en el marco de la globalización y sus desajustes y consecuencias.

El nuevo estilo nacional de desarrollo, en este plano económico-político, se ha caracterizado por el desarrollo de una desregulación de la economía, y por el abandono del Estado respecto de ciertos sectores estratégicos de la sociedad y de la política nacional, lo cual se demuestra con la reducción del gasto público, en el marco de las políticas de Ajuste Estructural, lo cual ha sido ampliamente abordado en diversos estudios e investigaciones (Korten, 1997). Por tanto, la gestión política del nuevo estilo nacional de desarrollo, y dentro de ella, la política educativa que incluye el tema de las condiciones laborales docentes, se opone a la propuesta de Kliksberg (1998), pues ha abandonado su orientación social y se ha reorientado hacia los criterios de empleabilidad o flexibilización laboral.

En este sentido la propuesta de Kilksberg (1998) aboga por lo que él denomina un Estado Inteligente, el cual define como un Estado con una política de Estado, no partidaria, con acciones claras en lo educativo, la cultura, la salud, promotor de una sociedad, que amalgame el crecimiento económico y el desarrollo social, atento a las consecuencias sociales económicas, y ambientales de la globalización, intermediario entre distintos actores sociales. Una forma de Estado que en Costa Rica, al menos desde la década de 1980 hasta la actualidad, no hemos logrado construir, lo cual ha tenido implicaciones profundas en las cuestiones educativas, y de una manera muy particular, ha incidido fuertemente en las condiciones laborales del sector docente.

Es en este contexto, característico del nuevo estilo nacional de desarrollo del país, iniciado en la década de 1980, y consolidado en la primera década del nuevo siglo, en el que se desarrolla la práctica profesional de la docencia, pero sobre todo, es en este contexto, 


\section{Gestión}

Revista Cientifica Digital ISSN-2215-2288

neoliberalmente condicionado, en el que se define la política educativa, y por tanto, las condiciones laborales de los docentes, las cuales analizaremos con más detalle en el siguiente apartado.

\section{Condiciones laborales para el sector docente en Costa Rica}

Bourdieu y Passeron (1996), han planteado que la estructura y funcionamiento de lo que ellos denominan Sistema de Enseñanza - es decir, del Sistema Educativo - están en función de la necesidad de producir y reproducir condiciones para su existencia misma, y para su función de reproducción social. Las condiciones para la existencia de un determinado Sistema de Enseñanza deben estar no solo aseguradas sino que, deben reproducirse, para ello todo Sistema de Enseñanza requiere un grupo de agentes especializados dotados de formación homogénea, instrumentos homogeneizados y homogeneizantes para realizar un Trabajo Pedagógico y un Trabajo Escolar específico y reglamentado.

El sistema de enseñanza impide el ejercicio del trabajo escolar heterogéneo mediante condiciones institucionales e incluso, mediante condiciones laborales específicas, cuyo deterioro, reduce la calidad de la educación en general y, por tanto, aumenta la reproducción de las condiciones sociales en las que se desarrolla el sistema educativo. El sistema de enseñanza produce una cultura escolar rutinizada y además, reproduce no solo arbitrariedad cultural por medio de la violencia simbólica, sino que también produce reproductores de arbitrariedad cultural; es decir, docentes que, en la mayoría de los casos, de manera inconsciente, trabajan en la reproducción de las condiciones de existencia de ese sistema, debido a las mismas condiciones laborales e institucionales que ese sistema les brinda (Morales, 2009).

El problema de las condiciones laborales de los y las docentes no está solo relacionado con su formación, si bien, el poco control sobre las carreras de formación docente, (sobre todo en la cantidad y calidad de programas ofrecidos por las Universidades Privadas que son las más) es un factor clave para la comprensión del estado de la profesión en la actualidad pues permite observar como la saturación del mercado laboral de mano de 
obra docente profesional ha hecho que las condiciones se deterioren para este sector, pues al aumentar la cantidad de oferta de este servicio, el precio con que se cotiza en el mercado este sector disminuye.

No existe en Costa Rica una política de Estado que regule la relación entre formación de docentes y mercado laboral, de una manera en que se planifique cualitativa y cuantitativamente la formación de profesionales en general, y docentes particularmente (Morales, 2011). Ni los sindicatos, ni el Estado, ni el Colegio de Licenciados y Profesores en Letras, Filosofía, Ciencias y Artes [COLYPRO] han mostrado interés en articular o negociar, ni aun pensar o sugerir una política de este tipo.

Tradicionalmente, uno de los principales temas de interés en lo que respecta a las condiciones laborales de los docentes es la cuestión salarial, pues siempre la reivindicación salarial está como una de las demandas recurrentes cuando se discute sobre el trabajo docente, pero en pocos casos se plantea que si bien este es un tema necesario, no es un tema suficiente para el abordaje integral de las políticas educativas en torno a las condiciones laborales de los docentes.

En el Tercer Informe sobre el Estado de la Educación (CONARE, 2011), cuando se plantea el estado de las condiciones laborales, se indica que uno de los hallazgos claves es el siguiente:

El ingreso promedio de los educadores mejoró significativamente en los últimos cinco años, como resultado de procesos de negociación con las organizaciones gremiales y de políticas del MEP. El objetivo fue equiparar los salarios de los profesionales del sector público central con aquellos del denominado percentil 50 correspondientes al sector público autónomo no financiero. Los salarios de los docentes pasaron del percentil 25 al percentil al percentil 50 en el 2010. (p. 144)

Como se aprecia en la cita anterior, hay varios elementos que deben ser analizados, y que no se pueden tomar a la ligera. El primero de ellos es el tema de la relación que se busca establecer de manera sutil entre mejora de las condiciones laborales-salariales y la gestión del Ministerio de Educación Pública dirigido en los últimos cinco años por 
Leonardo Garnier Rímolo. Es decir, se puede inferir una suerte de complacencia de parte del Informe, respecto de la política oficial del Partido Liberación Nacional en materia de educación, implementada durante los últimos cinco años por el señor Garnier.

Pero además de esa interpretación política sobre lo que plantea el Informe, no hay por qué suponer que de una mejora en los salarios se deriva, inmediatamente, una mejora de las condiciones laborales del sector docente. El tema salarial (planteado de manera simplista como aumento salarial) es un tema necesario pero es a la vez un tema insuficiente cuando se habla de las condiciones laborales de un sector específico de la estructura socioocupacional. Pues existen muchos otros elementos que deberían tenerse en cuenta cuando se plantean las condiciones laborales como elemento central de la política educativa de un país; elementos sobre los cuales volveremos más adelante. En la siguiente tabla se puede apreciar la mejora que el Tercer Informe sobre el Estado de la Educación plantea en materia salarial para las y los docentes:

\section{Tabla N. 1}

Salario base, según clase profesional de los docentes de centros educativos de enseñanza secundaria, en grupos seleccionados. 2004 y 2009

\begin{tabular}{|c|c|c|c|c|}
\hline \multirow[b]{2}{*}{ Colones corrientes } & & \multicolumn{3}{|c|}{ Profesor de enseñanza media } \\
\hline & & MT4 & MT5 & Posgrado \\
\hline 2004 & & 222.200 & 232.067 & 237.662 \\
\hline 2009 & & 503.400 & 565.800 & 581.333 \\
\hline \multicolumn{5}{|l|}{ Colones constantes } \\
\hline 2004 & & 299.667 & 312.974 & 320.527 \\
\hline 2009 & & 379.258 & 426.269 & 437.972 \\
\hline $\begin{array}{l}\text { Diferencia porcentual } \\
2009\end{array}$ & 2004- & 21,0 & 26,6 & 26,8 \\
\hline
\end{tabular}

Fuente: Tercer Informe sobre el Estado de la Educación, 2011.

Se puede apreciar que en efecto existe un aumento entre los años que van de 2004 a 2009. Sin embargo, este aumento es claramente relativo, pues parece, hasta cierto punto lógico, que en un lustro, los salarios deberían aumentar y además, parece lógico también 
que exista una equiparación con el resto de profesionales del sector público, con tal de brindar igualdad relativa en materia de condiciones laborales.

No obstante lo anterior, podríamos considerar, además, que el trabajo docente, a diferencia de otros trabajos del sector público, presenta una diferencia notable en términos de las jornadas de trabajo directo e indirecto. La docencia indirecta, esas horas que muchos docentes dedican a la revisión de cientos de exámenes, al planeamiento de las lecciones y a la confección de materiales didácticos, horas de trabajo en casa no consideradas en la retribución salarial, lo cual causa evidentemente desgaste laboral (Cordero, 2010), pues lo que se paga son las lecciones directas y algunos incentivos por zonaje, anualidades, más lo referente a los 200 días lectivos, privilegios que son propios (excepto los 200 días lectivos) de la administración pública.

Con respecto al tema salarial, el Ministerio de Educación Pública [MEP], indicó en su página web $^{5}$, el 26 de enero de 2009, en el momento en que se realizaron los aumentos salariales al sector docente, la siguiente información, a modo de ejemplo sobre el aumento salarial:

\section{Tabla N.2}

\section{Aumento Salarial}

\section{Docente bachiller de Secundaria Académica - Grupo profesional MT4}

Incluye salario 40 lecciones, Carrera Profesional 18 puntos y 5 Anualidades

\begin{tabular}{lll}
\hline Mayo 2006 & Enero 2009 & Diferencia \\
$\$ 356.935$ & $\$ 491.565$ & $\$ 134.630(+38 \%)$
\end{tabular}

Fuente: Elaboración propia con datos del sitio web del MEP

Evidentemente, se aprecia un aumento significativo, pero al mismo tiempo relativo, sobre todo por el tema antes mencionado del pago de la docencia directa e indirecta. Además, otro detalle aun más complejo y problemático en la discusión, tiene que ver con que este aumento, así como el resto de aumentos hechos al sector docente en todos los puestos, contempla el incremento en función de 40 lecciones, cierta carrera profesional y cinco anualidades, elementos que podrían ser considerados básicos, sin embargo, no 
podríamos en ningún momento suponer, que todos los docentes del país están nombrados por 40 lecciones, sino que, existen docentes nombrados por una cantidad de tiempo muy inferior a las 40 lecciones.

Otro problema importante en relación al tema salarial es el descontrol y la incapacidad del MEP para realizar el pago completo y puntual, tal como lo establece toda la jurisdicción existente en materia laboral, el cual es un tema que ha estado sin resolver por mucho tiempo, y que aún se mantiene vigente, tal como lo indica el profesor Ramírez (2011) que el Ministerio de Educación incumple el mandato constitucional de pago completo y puntual de los compañeros educadores, lo que les afecta de muchas maneras.

Esta situación del incumplimiento en el pago puntual e íntegro del salario es un problema que genera, junto con el resto de problemas antes mencionados en materia salarial, no solo incerteza laboral, sino frustración y, por lo tanto, desde el punto de vista de la sociología del trabajo, estos fenómenos tienden a ocasionar desvinculación y a crear identidades laborales frágiles; es decir, la identificación que se puede tener respecto del trabajo en estas condiciones es poca, por lo tanto, es probable que el compromiso con hacer el trabajo de la mejor manera posible, disminuya, pues la retribución primaria, la cual es el salario se ve desvirtuada (Dubar, 1992).

Estos fenómenos tienen una profunda incidencia sobre el gremio docente, pues hay una tendencia a considerar no solo que no es necesario comprometerse tanto con el trabajo que se hace, lo cual afecta directamente la calidad de la educación que recibirán los estudiantes que están a cargo de docentes desestimulados salarialmente, y además, hay una devaluación social de la labor docente (Deranty, 2008). Con estas condiciones, no podríamos imaginar si quiera, que en las jóvenes generaciones, individuos con altos perfiles culturales y académicos tiendan o quieran inclinarse por la profesión docente, pues se encuentra económica y socialmente desprestigiada o devaluada, dada la precarización de las condiciones laborales de este sector de la clase trabajadora (Molina, 2007), obviamente esto no es un determinismo, siempre existirán excepciones.

La configuración de este tipo de condiciones laborales es propia del sistema capitalista y de la sociedad burguesa; lo particular es que en nuestro país, la lógica haya 
salido de la explotación de la mano de obra no calificada, industrial y se esté desarrollando a lo interno del sistema educativo y del sector docente.

El actual orden económico capitalista es como un cosmos excepcional, en el que el individuo nace y al que, al menos en cuanto individuo, le es dado como un edificio prácticamente irreformable, en el que ha de vivir, y al que impone normas de su comportamiento económico, en cuanto que se halla implicado en la trama de la economía... El capitalismo actual, señor absoluto en la vida económica, educa y origina, por la vía de la selección económica, los sujetos, tanto empresarios como trabajadores, que necesita. (Weber, 2001, p.36)

Por tanto, tal como lo señala Weber (2001), coincidiendo con Bourdieu y Passeron (1996), en el análisis del sistema educativo, este sistema genera las condiciones para su reproducción y para convertirse en un verdadero establishment afianzado en las fuerzas de poder económico-político que dirigen el Estado, y por lo tanto, el desarrollo de la política educativa nacional. En estas condiciones, una lucha contra ese edificio irreformable que plantea Weber (2001), es no solo complicada, sino que, para muchos educadores resulta impensable en términos de cómo operacionalizar esa lucha, pues aun los sindicatos no han tenido hasta el momento la suficiente capacidad organizativa para articular una movilización que exija y logre revertir esta situación.

Cuando revisamos las condiciones laborales de los docentes, vemos que las fuertes reivindicaciones están en función, la mayoría de las veces, en lo referido al tema salarial, y además, en el caso de Costa Rica, a los problemas relacionados con el pago puntual e integro del salario. Son problemas básicos, y que a pesar de ello, no han logrado ser resueltos por las instancias competentes, y aunque parece ser que esta es una tendencia global del capitalismo de nuestra época, la cual se dirige al deterioro sistemático de las condiciones laborales, lo que algunos han llamado flexibilización de las condiciones laborales, empleabilidad o trabajo desregulado (De Filippis y McGrath, 2009), para el caso del sector docente del país, este deterioro trasciende el tema salarial. 
Por tanto, además del problema salarial, el tema del interinazgo es otro elemento que contribuye con el deterioro de las condiciones laborales de los y las educadoras de nuestro país, un tema señalado en el Tercer Informe sobre el Estado de la Educación (CONARE, 2011), al indicar que el problema persiste en el nombramiento de docentes, ya que casi la mitad están en esta condición.

Como bien lo anota el Informe (CONARE, 2011), este es un problema que sigue irresuelto en el sistema educativo nacional y para el cual no se visualiza, en este momento, una política de Estado dirigida a brindar una solución concreta y expedita. En la siguiente tabla se puede apreciar la cantidad de profesores interinos por nivel educativo entre 2002 y 2009:

\section{Tabla N.3}

Nombramientos de personal docente y docente-administrativo en propiedad por nivel en la educación pública tradicional

\begin{tabular}{cccccc} 
Nivel educativo & $\mathbf{2 0 0 2}$ & $\mathbf{2 0 0 5}$ & $\mathbf{2 0 0 7}$ & $\mathbf{2 0 0 8}$ & $\mathbf{2 0 0 9}$ \\
\hline Preescolar y primaria & 69,7 & 67,4 & 69,6 & 66,2 & 65,9 \\
Secundaria académica & 49,2 & 46,6 & 46,3 & 44,2 & 43,5 \\
Secundaria técnica & 45,6 & 40,6 & 43,2 & 40,4 & 42,5 \\
Educación especial & 46,6 & 45,6 & 50,8 & 45,9 & 47,3 \\
Educación de adultos & 18,2 & 21,7 & 23,1 & 22,0 & 21,6 \\
\hline Total & 59,0 & 55,4 & 57,2 & 54,1 & 53,8 \\
\hline
\end{tabular}

Fuente: Tercer Informe sobre el Estado de la Educación, 2011 


\section{Gestión:}

Revista Cientifica Digital ISSN-2215-2288

La tabla anterior es de sumo interés, pues permite observar como el interinazgo es un fenómeno transversal a todo el sistema educativo nacional, y además, se observa que es un problema constante, histórico, ya que en siete años, no se aprecia una disminución clara, producto de una política educativa sistemática que permita la reducción de esta condición laboral.

La situación de interino es un problema que genera incertidumbre en cuanto a las condiciones materiales de existencia, es decir, la incerteza en cuanto a la capacidad de obtener un trabajo estable, seguro, y con buenas condiciones laborales hace que los trabajadores de la educación se enfrenten directamente a lo que Beck (1998) y otros sociólogos europeos han llamado sociedad del riesgo. Esta condición de incertidumbre tiene efectos sobre la práctica profesional, y sobre el estado anímico del trabajador, pues es una condición poco estimulante al sentirse en un contexto inestable, o como Bauman (2003) lo ha denominado, un contexto propio de la modernidad líquida.

Ahora bien, es importante considerar también que esta condición de plazas vacantes, a pesar de la intervención de la Dirección General del Servicio Civil en los mecanismos de contratación del MEP, tiene todo el potencial para convertirse en una herramienta política, pues tal cantidad de plazas, representan un capital laboral de enorme peso en diversos tipos de negociaciones, o bien de otras situaciones irregulares que no han sido documentadas, pero que se relacionan con un anquilosado e histórico clientelismo político.

Hay un tema que pocas veces se toma en cuenta a la hora de discutir sobre las condiciones laborales que enfrentan los y las docentes en nuestro país, el cual tiene que ver con la cantidad de estudiantes que un docente atiende por grupo. Al respecto Ruiz (2006), indica que:

En los países de la OECD, por ejemplo, el tamaño promedio de las clases es 21-22 y en el tercer ciclo de unos 23-24 (OECD, 2002). En los países europeos el número es menor que el promedio, y en Japón y Corea es mucho mayor. El ejemplo de Cuba en su nueva reforma educativa, lo repetimos: máximos de 20 alumnos en primaria y 15 alumnos en el tercer. (p. 34) 
De modo que, en los países que han logrado consolidar sistemas educativos de alta calidad, el tema de la cantidad de estudiantes por grupo es fundamental, y se aprecia la capacidad de gestión de la educación en el sentido de lograr reducir el número de estudiantes, pues claramente esto incide positivamente sobre el sistema educativo y la capacidad de un docente de atender con metodologías menos generalizantes - u homogeneizantes, según Bourdieu y Passeron (1996) y más focalizadas para el desarrollo de los estudiantes con que trabaja.

En Costa Rica, el promedio de estudiantes por sección en el III ciclo y en la educación diversificada es de 30 estudiantes para el año 2009 (CONARE, 2011). Sin embargo, es común escuchar docentes que dan testimonio de trabajar con grupos de 40 o más estudiantes, lo cual incide directamente sobre la calidad del trabajo que puedan realizar, y sobre la recarga laboral que ello implica, en términos de atención, planeamiento de lecciones, confección y revisión de exámenes y otro tipo de trabajos.

Otro tema ausente en el análisis de las condiciones laborales del sector docente, sobre el cual existen pocos datos, y además no hay investigación al respecto, es la cantidad de docentes que con tal de acceder a una plaza en propiedad en el Ministerio de Educación Pública [MEP], deben desplazarse a lugares sumamente alejados de su residencia, creando una serie de problemáticas individuales importantes.

El hecho de desplazarse a un lugar en extremo lejano de la residencia, implica que una serie de vínculos familiares, afectivos, comunitarios, y otros, se vean afectados, lo cual tiene directamente implicaciones sobre el estado anímico de los docentes, y por tanto sobre el trabajo que realizan. Además, esto genera también una identidad laboral instrumental, es decir, el trabajo se hace únicamente por la retribución salarial y la estabilidad laboral que se puede lograr, por lo que en muy pocos casos se desarrollan vínculos importantes con la comunidad receptora, y muy por el contrario, en la medida de lo posible se trata de regresar a la zona de origen cuanto antes, sobre todo si se cuenta con una familia, hijos, u otras personas de una muy cercana relación de parentesco, o que dependen del trabajador.

Sin embargo, este es un tema que está pendiente de ser investigado, pues ni el Informe sobre el Estado de la Educación en sus tres entregas (2005, 2008, y 2011) se ha 
ocupado del tema, y tampoco la investigación académica, al menos en la Universidad de Costa Rica lo ha hecho. Sería interesante, recabar en una futura investigación, las experiencias de docentes que paradójicamente, han tenido que desplazarse para alcanzar una plaza, lo cual implica todo un tema de contratación, de planificación en la formación de educadores y de política laboral y educativa a tanto a nivel nacional, regional y local.

Existe también el país, una política de desregulación del mercado de bienes educativos, propia de la concepción mercantil de la educación en un contexto condicionado por el neoliberalismo, lo cual ha llevado a la creación desmesurada de carreras de educación, lo cual incide directamente sobre la calidad de la educación en el país, y además, sobre las condiciones laborales del sector docente. De manera que, tal como lo apunta el Tercer Informe sobre el Estado de la Educación:

No existe un dato oficial del número de carreras que ofrece el conjunto de universidades del país. El CONARE elabora una aproximación sobre la distribución de programas según instituciones con base en la información que le suministran las universidades públicas directamente, y las universidades privadas a través del CONESUP. Esta estimación entiende por “oportunidad académica” toda carrera autorizada que cumple con lo establecido en el "Fluxograma de creación de nuevas carreras y de modificación de las ya existentes”. (CONARE, 2011, p.167)

La cantidad de oportunidades académicas en el campo educativo y en el campo de las ciencias sociales y económicas es de una gran amplitud, lo cual evidencia la falta de interés de parte de los grupos que han accedido al poder del Estado, por desarrollar una política educativa integral, que tome en cuenta la relación entre mercado laboral, y formación docente. En la siguiente tabla podemos apreciar la cantidad de oportunidades académicas:

\section{Tabla N.4}


Oportunidades académicas por sector, según área de estudio, 2010

\begin{tabular}{lcccccc}
\hline & \multicolumn{2}{c}{$\begin{array}{c}\text { Universidades } \\
\text { públicas }\end{array}$} & \multicolumn{2}{c}{$\begin{array}{c}\text { Universidades } \\
\text { privadas }\end{array}$} & \multicolumn{2}{c}{$\begin{array}{c}\text { Total de } \\
\text { universidades }\end{array}$} \\
\hline Área & Total & Porcentaje & Total & Porcentaje & Total & Porcentaje \\
\hline Artes y Letras & 55 & 9,1 & 31 & 5,8 & 86 & 7,6 \\
Ciencias Básicas & 29 & 4,8 & & & 29 & 2,5 \\
Ciencias de la Salud & 106 & 17,5 & 45 & 8,4 & 151 & 13,3 \\
Ciencias Económicas & 69 & 11,4 & 137 & 25,6 & 206 & 18,1 \\
Ciencias Sociales & 92 & 15,2 & 113 & 21,1 & 205 & 18,0 \\
Computación & 32 & 5,3 & 25 & 4,7 & 57 & 5,0 \\
Educación & 120 & 19,9 & 130 & 24,3 & 250 & 21,9 \\
Ingeniería & 55 & 9,1 & 49 & 9,2 & 104 & 9,1 \\
Recursos Naturales & 46 & 7,6 & 5 & 0,9 & 51 & 4,5 \\
\hline Total & $\mathbf{6 0 4}$ & $\mathbf{1 0 0 , 0}$ & $\mathbf{5 3 5}$ & $\mathbf{1 0 0 , 0}$ & $\mathbf{1 1 3 9}$ & $\mathbf{1 0 0 . 0}$ \\
\hline
\end{tabular}

Fuente: Tercer Informe sobre el Estado de la Educación, 2011.

Como se puede observar, el total de oportunidades académicas en el área de educación es de 250, la más alta de todas, lo cual, aunado a los pocos criterios de ingreso a las carreras de formación docente que existen en las universidades tanto públicas como privadas, hace que exista un aumento cuantitativo en la formación de docentes, pero al mismo tiempo, saltan cuestionamientos sobre la calidad de esta formación. Además, resulta ilógica, la relación entre titulación y necesidades del mercado laboral, siendo otra de las consecuencias el desempleo y la subutilización del recurso profesional docente, tema que en el Tercer Informe sobre el Estado de la Educación (CONARE, 2011), se encuentra ausente, y que además no hay datos para este sector específico de la fuerza laboral.

Esta dinámica ha llevado a un deterioro o precarización de las condiciones laborales, pues al existir sobre oferta de mano de obra profesional en algunos campos específicos de la estructura socio-ocupacional, deriva en una devaluación social y económica de algunas profesiones en particular, y además, existe una política de Estado propia de la desregulación laboral o flexibilización que en términos generales ha sido estudiada por Trejos (1997) y que, para el caso de ciencias sociales, ha sido ampliamente analizado por el estudio de Villagra (2010); además, por el trabajo de Mora (2005), pero para el caso de los trabajadores docentes aun falta más investigación académica al respecto (referidos por Morales, 2011). 


\section{Consideraciones finales}

Es evidente que existen problemas que se han convertido en tendencias históricas dentro del desarrollo de la profesión docente en nuestro país, sobre todo el tema salarial (considerado íntegramente, no solo en cuanto al monto del salario, sino también en el pago completo y puntual) y el tema del interinazgo, así como otros problemas mencionados y analizados anteriormente, relacionados con el desplazamiento de educadores a zonas alejadas, y el recargo laboral (Cordero, 2010) en funciones tanto docentes como de otro tipo.

Sin embargo, luego de observar de una manera panorámica y general la política y las condiciones para los y las docentes en Costa Rica, es notable la inexistencia de un programa político sistemático que esté orientado a mejorar gradualmente los problemas que este sector de la clase trabajadora enfrenta.

Muy por el contrario, fuera de los aumentos salariales, más bien parece que la lógica de las condiciones políticas buscadas por los grupos que controlan el poder del Estado está orientada hacia la desregulación, la flexibilización laboral o empleabilidad, lo cual deriva en una precarización de las condiciones laborales, fenómeno iniciado junto con el proceso de liberalización de la economía del país, (es decir con la firma de los Programas de Ajuste Estructural y el Tratado de Libre Comercio con EE.UU) y un conjunto de leyes que institucionalizan la flexibilización (Carballo, 2010) cuyo intento de consolidación de esas condiciones, ha sido el proyecto de Ley propuesto por el MIDEPLAN bajo el número de expediente 17628, llamado Proyecto de ley general de la relación publica de servicio.

Por lo tanto, no es posible coincidir, bajo ninguna circunstancia con una de las conclusiones generales y principales del Tercer Informe sobre el Estado de la Educación (CONARE, 2011), la cual indica que en el sistema educativo costarricense ha tenido cambios positivos en los últimos cinco años que enriquecen no solo la educación pública sino la educación general, a nivel nacional.

Nótese la legitimación de la política educativa de los últimos cinco años, es decir, de los dos últimos gobiernos del Partido Liberación Nacional. No obstante, no podríamos decir, a la ligera, que vamos en la dirección correcta sin tomar en cuenta las condiciones 
laborales de los docentes del país, y plantear que hemos salido del estancamiento de las últimas décadas, cuando en realidad persisten viejos problemas como el interinazgo histórico. Además, no tenemos porque suponer, que el aumento salarial al sector docente, es lo más importante en una política laboral para la mejora de las condiciones de trabajo, y por lo tanto, para la mejora de la calidad de la educación.

Por otro lado, consideramos importante resaltar con Bourdieu y Passeron (1996) que el trabajo pedagógico produce habitus conforme a la arbitrariedad cultural que representa, y es necesario recordar también, que el habitus produce prácticas sociales, las cuales en este caso estarán por lo tanto en función de la arbitrariedad cultural que se representa. La efectividad del trabajo pedagógico se mide en el tanto produce su efecto de reproducción de la arbitrariedad cultural y por lo tanto de la Estructura Social, también la efectividad del trabajo pedagógico se mide en el tanto el habitus que reproduce es duradero y transferible a otros aspectos de la vida.

Lo anterior, quiere decir que dadas ciertas condiciones sociales, políticas, económicas y, por lo tanto, laborales en las que se desarrolla el trabajo pedagógico, el trabajo docente es posible que se llegue a una naturalización de esas condiciones, y no se comprenda que son una construcción socio-histórica y socio-política, lo cual conduce a la imposibilidad de pensar en la transformación de esas condiciones.

Pero más grave aún, esa naturalización del habitus que produce el trabajo docente, y el trabajo en general en las sociedades capitalistas, puede llevar incluso a la aceptación consciente o inconsciente, de las condiciones laborales flexibles, desreguladas y precarias, como algo deseable o algo a lo que se aspira, pues en primera instancia es necesario obtener un trabajo, para solventar las necesidades más elementales de los individuos y sus familias, y si ese trabajo tiene unas condiciones laborales naturalizadas, aun siendo precarias, puede que estas condiciones sean aceptadas y deseadas, pues es lo que hay.

Tampoco se aprecia que este sector de trabajadores y trabajadoras tengan una capacidad de movilización y articulación que le permita negociar en bloque sus condiciones laborales, de nuevo, más allá de lo estrictamente salarial. Esto puede estar relacionado con el bajo estatus social que en Costa Rica posee la profesión docente, y además con la desidentificación que se muestra respecto de la profesión, pues hay una tendencia a la 
configuración de identidades laborales frágiles, instrumentales, y flexibles, esto es, con poca identificación y con interés únicamente en la retribución económica, propio de la mercantilización neoliberal de la educación.

Es importante enfatizar que no es posible, (ni lo será) hablar de calidad de la educación, y de gestión del cambio en el sistema educativo, si lo único que se plantea en términos de las condiciones laborales de los y las docentes, es el tema del aumento salarial. La calidad de la educación requiere de una abordaje integral de las condiciones laborales, en el tanto, no se realice una revisión a fondo de la política laboral que el Estado define en torno a este sector de la clase trabajadora, la educación nacional, lejos de avanzar, continuara retrocediendo en amplios y diversos aspectos.

\footnotetext{
Notas

${ }^{1}$ La Investigación legitimante, plantea J.L. Coraggio, es aquella que tiene por objetivo el asegurar la aceptación de las políticas vigentes, sobre todo cuando dichas políticas han sido puestas en entre dicho por un nuevo paradigma emergente, siendo la diferencia para el caso costarricense, el hecho de que no existe en este momento un paradigma emergente que ponga en entredicho la política educativa desarrollada por las administraciones 2006-2010 y 2010-2014. Y el Informe es legitimante por que dentro de sus conclusiones sobresale que en materia de educación vamos por buen camino, sobre todo desde los últimos cinco años (CONARE, 2011).

${ }^{2}$ La Investigación legitimante, plantea J.L. Coraggio, es aquella que tiene por objetivo el asegurar la aceptación de las políticas vigentes, sobre todo cuando dichas políticas han sido puestas en entre dicho por un nuevo paradigma emergente, siendo la diferencia para el caso costarricense, el hecho de que no existe en este momento un paradigma emergente que ponga en entredicho la política educativa desarrollada por las administraciones 2006-2010 y 2010-2014. Y el Informe es legitimante por que dentro de sus conclusiones sobresale que en materia de educación vamos por buen camino, sobre todo desde los últimos cinco años (CONARE, 2011).

${ }^{3}$ El concepto de estilo nacional de desarrollo ha sido utilizado por Jorge Rovira Mas - quien a su vez lo ha tomado de Jorge Graciarena- para referirse al movimiento que realiza una sociedad en un momento histórico-social determinado, movimiento que se da en un marco de factores condicionantes externos (la dinámica política, económica, social y cultural internacional), pero que debe su dirección, su ritmo, y orientación a los factores determinantes internos (los actores y las fuerzas políticas que se enfrentan entre si por imponer o llevar a cabo una concepción económica, política y social particular). Este concepto posee una gran utilidad para captar el movimiento de una sociedad, y sobre todo para observar el estado de un colectivo nacional como una resultante histórica producto de la interacción de distintos fenómenos externos e internos.

${ }^{4}$ Esto salta a la vista en la discusión sobre el tratado de libre comercio con Estados Unidos, la coalición del SI era, a pesar de sus divisiones, un movimiento político coherente, con un proyecto claramente definido, concreto, mostrando que a pesar de la heterogeneidad del sector político-económico, tenían la capacidad de dar una lucha por una causa común. Por otro lado, el movimiento del NO, fue lo contrario, tuvo un alto nivel de fragmentación, no era un proyecto político, era una resistencia, y bajo una misma causa se encontraban sectores que en torno a otros temas serían totalmente contrarios, por ejemplo, algunos sectores de la Iglesia Católica, junto a sectores defensores de la diversidad sexual. Para un análisis detallado
} 
de las coaliciones del SI y del NO, en el contexto de la discusión del TLC, la investigación de Torres Vindas (2010) es un referente muy interesante.

${ }^{5}$ www.mep.go.cr

\section{Referencias Bibliográficas}

Araya, H., Barrantes, I., Vega, J. (2011). Un análisis crítico del estado de la investigación educativa sobre exclusión del sistema educativo formal, publicada en el periodo 2000-2009. Ponencia presentada en el II Congreso Internacional de Investigación Educativa. Instituto de Investigación Educativa. Universidad de Costa Rica. San José, Costa Rica.

Bauman, Z. (2003). Modernidad líquida. Argentina: Editorial Fondo de Cultura Económica.

Beck, U. (1998). La sociedad del riesgo. Hacia una nueva modernidad. Barcelona: Editorial Paidós.

Bourdieu, P., Passeron, J.C. (1996). La Reproducción: elementos para una teoría del sistema educativo. México: Fontamara.

Bourdieu, P., Passeron, J.C. (2004). Los Herederos: los estudiantes y la cultura. Argentina: Siglo veintiuno editores.

Braslavsky, C., Cosse, G. (1997). Las actuales reformas educativas en América latina: cuatro actores, tres lógicas y ocho tensiones. Programa de Promoción de la Reforma Educativa en América Latina y el Caribe. Revista Iberoamericana sobre Calidad, Eficiencia y Cambio en Educación [REICE], 4 (2). Recuperado de http://www.rinace.net/arts/vol4num2e/art1.htm

Carballo V., P. (2010). Mercado de trabajo precarizado dentro del Estado costarricense: las nuevas formas de contratación por tiempo definido para profesionales de ciencia sociales. Revista Centroamericana de Ciencias Sociales, 7 (1). San José, Costa Rica: FLASCO.

Consejo Nacional de Rectores [CONARE]. (2008). Segundo Informe sobre el Estado de la educación. Programa Estado de la Nación. San José, Costa Rica: CONARE.

Consejo Nacional de Rectores [CONARE]. (2011). Tercer Informe sobre el Estado de la educación. Programa Estado de la Nación. San José, Costa Rica: CONARE.

Coraggio, J. L. (1998). Sobre la investigación y su relación con los paradigmas 
Educativos. Novas políticas educacionais: Críticas e perspectivas. Recuperado de http:/hcdsc.gov.ar/biblioteca/ISES/educacion/A\%20119\%20\%20Investigacion\%20y \%20paradigmnas\%20educativos.pdf.

Cordero C., R. (2010). Análisis de la funciones y valoración de la carga laboral del puesto docente a nivel de primaria, de la agremiadas y los agremiados de la Asociación Nacional de educadores (ANDE).Instituto de Investigación en Educación [INIE]. Universidad de Costa Rica. San José, Costa Rica.

Cuvardic G., D. (2005). Construcción y representación fotográfica de los conflictos sociopolíticos: la 'huelga de educadores' del 2003 en el diario la nación. Revista Ciencias Sociales, 4 (106), 93-106. Instituto de Investigaciones Sociales. Universidad de Costa Rica. San José, Costa Rica.

De Filippis, J., McGrath, S. (2009). Social reproduction as unregulated work. Work, employment and society, BSA Publications, 23(1), 66-83. Los Angeles, London, New Delhi, Singapore and Washington DC.

Deranty, J. P. (2008). Work and the Precarisation of Existence. European Journal of Social Theory, 11 (4). Los Angeles, London, New Delhi and Singapore.

Dubar, C. (1992). Formes identitaires et socialization profesionelle. Revue Française de Sociologie, Année, Volume 33, Número 4, Paris.

Dubet, F. (julio, 2003). A escola e a exclusão. Cadernos de Pesquisa, 119, 29-45. Brasil.

Korten, A. (1997). Ajuste estructural en Costa Rica: una medicina amarga. San José, Costa Rica: Editorial DEI.

Lee, C. (2009). Critiques Historical Evolution of Risk and Equity: interdisciplinary Issues and Critiques. Review of Research in Education, 33, 63 - 100. Northwestern.

Milliband, R. (1985). El Estado en la sociedad capitalista. México: Editorial siglo XXI.

Molina J., I. (2007). Educación y sociedad en Costa Rica: de 1821 al presente (una historia no autorizada). Ponencia preparada para el Segundo Informe Estado de la Educación. Programa Estado de la Nación. San José, Costa Rica: CONARE.

Mora S., M. (2005). Ajuste y empleo: notas sobre la precarización del empleo asalariado. Revista de Ciencias Sociales, 108 (2). Instituto de Investigaciones Sociales. Universidad de Costa Rica. San José, Costa Rica. 
Morales Z., L. C. (2009). Durkheim y Bourdieu: reflexiones sobre educación. Revista Reflexiones, 88 (1). Facultad de Ciencias Sociales. Universidad de Costa Rica. San José, Costa Rica.

Morales Z., L. C. (2010). Balance de la sociología de la educación en Costa Rica. Revista Reflexiones, 89 (1). Facultad de Ciencias Sociales. Universidad de Costa Rica. San José, Costa Rica.

Morales Z., L. C. (2010). Cambios en el estilo nacional de desarrollo y promoción del sector turístico en Costa Rica. Revista de Ciencias Económicas, 28 (2). Universidad de Costa Rica. San José, Costa Rica.

Morales Z., L. C. (2011). Mercado laboral, educación superior y formación de docentes en Costa Rica. Revista Actualidades Investigativas en Educación, 11 (1). Instituto de Investigación en Educación [INIE]. Universidad de Costa Rica.

North, D. (setiembre, 1994). La nueva economía institucional y el desarrollo. Serie diálogo, 6. México.

Ramirez, R. (2011). Una vez más... Sección: Generando debate y conciencia. Recuperado de http://www.apse.or.cr/webapse/2011/deb/deb02.htm

Rovira M., J. (2004). El nuevo estilo nacional de desarrollo de Costa Rica 1984-2003 y el TLC. En Florez-Estrada, M. y Hernández, G. (Eds.). TLC con Estados Unidos. Contribuciones para el debate. ¿Debe Costa Rica aprobarlo? San José, Costa Rica: Instituto de Investigaciones Sociales, Universidad de Costa Rica.

Ruiz Z., Á. (2006). Universalización de la Educación Secundaria y Reforma Educativa. San José, Costa Rica: Editorial de la Universidad de Costa Rica-Consejo Nacional de Rectores [CONARE].

Trejos, M. E. (1997). Relaciones laborales y reestructuración del Estado en Costa Rica. San José, Costa Rica: Editorial Fundación UNA.

Torres V., J. (2010). Costa Rica 2007: Referéndum sobre el TLC. Las fuerzas sociopolíticas del SI y el NO frente al memorándum Casas-Sánchez. Tesis para optar al grado de Magister Scientiae en Sociología. Universidad de Costa Rica. San José, Costa Rica.

Vargas S., L. P. (2003). La estrategia de liberalización económica: 1980 - 2000. Cuadernos de Historia de las Instituciones. Universidad de Costa Rica. San José, Costa Rica. 
Venegas, M. E. (2010). Retos y desafíos de Costa Rica en materia de actualización profesional de los docentes de primaria y secundaria. Ponencia preparada para el Tercer Informe Estado de la Educación. Programa Estado de la Nación. San José, Costa Rica: CONARE.

Wacquant, L. (2006). Pensamiento crítico y disolución de la doxa. Revista ANTIPODA, 2, 43-51. Universidad de los Andes. Colombia. Recu http://antipoda.uniandes.edu.co/view.php/17/index.php?id=17 perado de

Weber, M. (2001). La ética protestante y el espíritu del capitalismo. Madrid: Mestas Ediciones.

Wright, M.C. (1994). La imaginación sociológica. México: Fondo de Cultura Económica. 